\title{
THEMATIC PAPER \\ Improvement work in mental healthcare: an example from Region Jönköping County, Sweden
}

\author{
Axel Ros, ${ }^{1} \odot$ Anna Österström, ${ }^{2}$ Göran Henriks ${ }^{3}$ and Boel Andersson-Gäre ${ }^{4}$
}

${ }^{1}$ Jönköping Academy for Improvement of Health and Welfare, School of Health and Welfare, Jönköping University, Sweden.Email:axel.ros@rilse

${ }^{2}$ Head of Quality Improvement, Division of Psychiatry and Rehabilitation, Region Jönköping County, Sweden

${ }^{3}$ Chief Executive of Learning and Innovation, Region Jönköping County, Sweden

${ }^{4}$ Jönköping Academy for Improvement of Health and Welfare, School of Health and Welfare, Jönköping University, Sweden

Keywords. Improvement work psychiatry; mental health; home treatment teams; quality.

First received 26 Feb 2020 Final revision 28 May 2020 Accepted 1 Jun 2020

doi:10.1192/bji.2020.37

(c) The Author(s) 2020. This is a Open Access article, distributed under the terms of the Creative Commons AttributionNonCommercial-NoDerivatives Coms. org/licenses/by-nc-nd/4.0/), which permits non-commercial re-use, distribution, and reproduction in any medium, provided the original work is unaltered and is properly cited. The written perproperly cisted. The written permission of Cambridge University Press must be obtained for commercial re-use or in order to create a derivative work.

\begin{abstract}
Region Jönköping County (RJC) in Sweden is a healthcare system that is characterised by sustainable work with quality in healthcare and long-term system-wide improvement. This article describes important factors behind the improvement work in RJC, and how the improvement methods and initiatives have been adopted also in mental healthcare. For example, patients otherwise eligible for admission to a psychiatric department were treated at home after introduction of home treatment teams. Patient satisfaction was high and the number of visits to the emergency department, hospital admissions and hospital stay decreased.
\end{abstract}

\section{Region Jönköping County healthcare system}

Region Jönköping County (RJC) provides healthcare to the 365000 inhabitants of Jönköping County in southern Sweden at 3 hospitals and 40 primary care clinics. The primary care clinics provide the primary mental healthcare in their catchment areas. The departments of psychiatry at the three hospitals, with their in-patient wards and outpatient clinics, provide specialised psychiatric care; they work closely together at the regional level.

\section{The quality improvement approach in Region Jönköping County}

The starting point for RJC's quality journey was the early 1990s, when its management took on a 'quality as strategy' approach when the healthcare sector in Sweden was under pressure because of a national economic crisis. ${ }^{1}$ This approach has several important characteristics. ${ }^{2}$

We use a population health approach, based on the understanding of the correlation between experience of care, population health and per capita cost. ${ }^{3}$ Both the management and evaluation of the service embrace and support patient experience, innovation and learning, in addition to clinical results and cost-effectiveness.

Our view is that all healthcare is co-produced and co-designed with the patients and their families, ${ }^{4}$ therefore a focus on what is important for the patients and their families is key. We have developed 'peers', who are experienced patients, who engage the psychiatry departments to support other patients in improvement work. We also use exemplar personas such as 'Esther' or 'Britt-Marie', who represent various inhabitants in
Jönköping County. For example, the question 'What is good for Esther (or Britt-Marie and her family)?' leads the design and improvement work in RJC.

We provide education on improvement methods to leaders at all levels and to healthcare staff - another crucial aspect of our improvement work. Qulturum, the improvement and innovation centre of RJC, is staffed by improvement leaders, who support improvement work across $\mathrm{RJC}^{5}$ (Box 1). The Jönköping Academy for Improvement of Health and Welfare supports education and research as parts of the quality strategy. The Academy is a centre for education and research in management and improvement in the health and welfare sector established by RJC in cooperation with the 13 municipalities and Jönköping University.

The microsystem concept (where patients, families, care teams and data work together to improve care) is used and is widely taught to staff to support the continuous improvement work at the sharp end: a crucial concept in RJC quality management. ${ }^{6}$ Continuous nonhierarchical dialogues are used for feedback and learning following improvement work, between each level of the system; this is an important mechanism for sustaining the culture.

\section{Improvement work in mental healthcare} Improvement methods and initiatives have also been adopted in mental healthcare in RJC, illustrated here by an example from specialised psychiatric care.

\section{Psychiatric home treatment teams}

Home treatment teams have been established to provide specialised psychiatric care in the patient's home as a part of a community-based psychiatric service. A local trigger for the initiative was positive experiences in a nearby healthcare region and in one of our local departments that had already established mobile teams. Our goal was to move in-patient care, when judged safe and possible, to out-patient-oriented work with increased proximity and flexibility. The value and benefit to the patient are presumed to be a more person-centred care with increased continuity and safety by reducing: duration of hospital care, avoidable hospital admission and avoidable readmissions. Eligible patients are those in need of specialised psychiatric care who would otherwise have been admitted, or patients who are in 
Box 1. Methods and programmes that have been/are taught at Qulturum

- Clinical microsystems

- Improvement tools such as plan-do-study-act (PDSA), fish-bone diagrams and driver diagrams

- Leadership for improvement - for leaders at all levels

- Patient safety for management teams

- Access programmes

- In collaboration with Jönköping Academy, courses in:

- leadership for improvement

- patient safety

- co-production/co-design

Websites for more information:

Qulturum: https://plus.rjl.se/qulturum

Jönköping Academy for Improvement of Health and Welfare: https://center.hj.se/ jonkoping-academy/om-oss.html

hospital care where an earlier discharge is possible if care can be provided in their homes.

One specific aim was to reduce in-patient care and prevent hospital admissions by strengthening the out-patient care. Earlier studies have shown that specialised psychiatric home care by mobile teams can improve care, for example by increasing patient satisfaction, reducing stigma connected to psychiatric admission and reducing acute in-patient admissions by $30 \%{ }^{7}$

First, a multiprofessional regional quality improvement (QI) team, as well as local implementation teams in each department of psychiatry, were established. Peers, experienced patients and a skilled improvement advisor were also part of the QI team. Initially, they started by analysing the current situation in the different departments. Patients and different professionals were interviewed to identify gaps in needs and expectations, but also about new ideas on how to improve the care provided. The team studied existing mobile psychiatric teams in other healthcare regions. Several improvement tools were used by the QI team to sort and prioritise between actions, such as fish bone diagrams and driver diagrams before setting up a project plan, an action plan and a communication $\operatorname{plan}^{6,8}$ (Fig. 1). Small-scale tests according to the plan-do-study-act method were set up and evaluated continuously (weekly by the local implementation team and monthly by the regional QI team).

The concept of home treatment teams was developed across the three departments of psychiatry, but the local implementations were developed separately according to the local context. Some differences in staffing and services were accepted.

Care provided by the new home treatment teams includes: medication management, supportive conversation, anxiety management, massage, and education for patients and next of kin. Most of the teams are staffed by assistant nurses and nurses specialised in psychiatry, but other professionals, including psychiatrists, are available if necessary.

A preliminary evaluation of the first 45 patients included at one hospital has been
The goal

Primary drivers

'What?'
Secondary drivers

'How?/Actions'

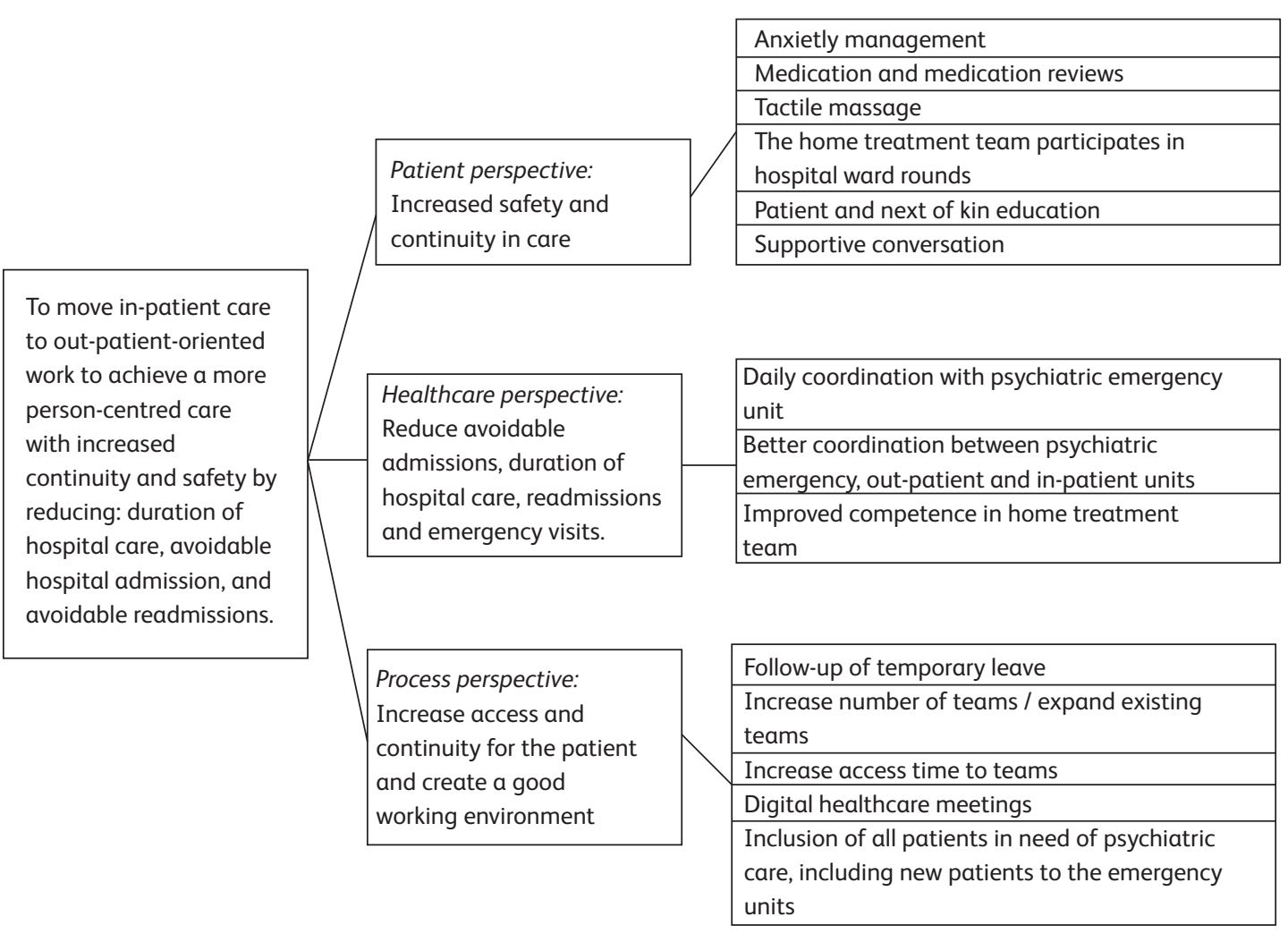

Fig. 1

Driver diagram for the implementation of home treatment teams. A driver diagram is a visual display of a team's theory of what 'drives', or contributes to, the achievement of a project aim and is a tool for communicating with a range of stakeholders where a team is testing and working. ${ }^{8}$ 
made. Visits to the emergency department per patient in the 90 days after support from the home treatment team was initiated compared with the 90 days before decreased (number of visits after support initiated, median (range): $1(0-6)$ $v$. before, 1 (0-8)). Hospital admissions per patient after support was initiated also decreased (after, $0(0-7) v$. before, $1(0-11))$, as did mean hospital stay per patient after support was initiated (after, $0(0-57)$ days $v$. before, $9(0-52)$ days). All differences are significant $(\alpha<0.01$, two-sided test, Wilcoxon signed-rank test). In a survey, patient satisfaction was high.

According to a survey among staff the establishment of the home teams led to a higher focus on and understanding of the patient's needs and abilities, but also increased interaction with next of kin. Home visits had also contributed to the identification of problems in transitions between hospital and home. Recognising these deficiencies contributed to valuable learning for improvement, as it allowed problems to be addressed at an earlier stage.

\section{Reflections on improvement in mental health}

RJC's 'quality as strategy' management principle is used in all improvement work across mental healthcare: access to treatment, patient involvement, reducing the need for hospital admission, organisational development of teams, and many others areas. Using this approach to improvement has led RJC to be recognised for its ongoing work with quality in healthcare and for its successful long-term, system-wide improvement. In several national comparisons over the years, the RJC stands out as one of the top healthcare systems in Sweden for clinical results and quality, patient satisfaction, public trust in the healthcare system and financial results.

In our experience, the improvement methods used for physical healthcare can be successfully employed in mental healthcare. This has also contributed to an insight that measurements of effects of improvement efforts are possible and necessary also in mental healthcare. The challenges that remain include: strategies to identify the patient's needs more accurately, difficulties in understanding how different healthcare professionals can contribute, language barriers - to be able to care for non-Swedish-speaking patients. We aim to further develop our culture of co-producing care across the whole system, including patients and next of kin in the co-design of all our improvement activities.

\section{Author contributions}

All four authors contributed to the conception of the article. A.R. and A.Ö. drafted the first versions of the article, all four authors then revised it and gave input to its final version. A.R. finalised the article, which was the finally approved by the other authors. All four authors agree to be accountable for all aspects of the work.

\section{Declaration of interest}

None.

ICMJE forms are in the supplementary material, available online at https://doi.org/10.1192/bji.2020.37.

\section{References}

1 Andersson-Gäre B, Neuhauser D. The health care quality journey of Jönköping County Council, Sweden. Q Manage Health Care 2007; 16: 2-9.

2 Övretveit J, Staines A. Sustained improvement? Findings from an independent case study of the Jönköping Quality Program. $Q$ Manage Health Care 2007; 16: 68-83.

3 Berwick DM, Nolan TW, Whittington J. The triple aim: care, health, and cost. Health Aff 2008; 27: 759-69.

4 Batalden M, Batalden P, Margolis P, Seid M, Armstrong G, Opipari-Arrigan L, et al Coproduction of healthcare service. BMJ Qual Saf 2016; 25: 509-17.

5 Bodenheimer T, Bojestig M, Henriks G. Making systemwide improvements in health care: lessons from Jönköping County, Sweden. Q Manage Health Care 2007; 16: 10-5.

6 Nelson EC, Batalden PB, Godfrey M. Quality by Design: A Clinical Microsystems Approach. John Wiley \& Sons, 2011.

7 Johnson S. Crisis resolution and home treatment teams: an evolving model. Adv Psychiatr Treat 2013; 19: 115-23.

8 Institute for Healthcare Improvement. Driver Diagram. Institute for Healthcare Improvement, 2020 (http://www.ihi.org/resources/ Pages/Tools/Driver-Diagram.aspx)

\title{
THEMATIC PAPER \\ Reducing the risk of patient suicide in Tuscany
}

\author{
Giulia Dagliana, ${ }^{1}$ Sara Albolino, ${ }^{2}$ Laura Belloni ${ }^{3}$ and Tommaso Bellandi ${ }^{4}$
}

${ }^{1} \mathrm{MSc}$, Safety and Quality Manager, Coordinator WHO Collaborating Centre in Human Factor and Communication for the Delivery of Safe and Quality Care, Florence, Italy

${ }^{2}$ PhD-EurErg, Director, Centre for Clinical Risk Management and Patient Safety, Florence, Italy

${ }^{3} \mathrm{MD}$, Psychiatrist, Director of the
Patient suicide is one of the most frequent incidents in healthcare facilities to be reported to the National Observatory of Sentinel Events in Italy. Despite national initiatives, in Tuscany potentially preventable patient suicides still occur in both acute and community care settings. We describe here an aggregated qualitative analysis of 14 patient suicides that took place in public health services between 2017 and 2018. We outline the methodology and results of an improvement action we enacted in the healthcare system that involved reviewing and reinforcing relevant managerial strategies and clinical activities, with the aim of reducing potentially preventable patient suicides. 